\title{
Efficient Multi-Hop Communications in Bluetooth Scatternets
}

\author{
Liza Lai-Yee Shek and Yu-Kwong Kwok \\ Department of Electrical and Electronic Engineering \\ The University of Hong Kong, Pokfulam Road, Hong Kong,China
}

\begin{abstract}
This study proposes an integrated ad hoc routing and time-slot scheduling (IARTSS) scheme to address the problem of ad hoc routing in Bluetooth networks. Our proposed scheme contains four main mechanisms to address the different facets of the problem, namely CompensationBased Time-Slot Assignment (CTSA), Traffic Differentiation Queueing (TDQ), Adaptive Master-Slave Switching (AMSS), and an Enhanced AODV algorithm for ad hoc routing. CTSA judiciously allocates time slots to slaves based on elapsed time, utilization, and queue lengths, helping the bridge nodes to catch up with the lagging of services in piconets. TDQ differentiates traffic into self-originated and forwarded messages, and serves them in a dynamically adjusted adaptive ratio. AMSS calculates the time for a bridge node to stay in a piconet in a more effective way, based on utilization fraction and queue lengths. Enhanced AODV for ad hoc Routing is implemented as a routing protocol for Bluetooth scatternet. We have built a comprehensive Bluetooth simulator and performed extensive simulations to evaluate the proposed IARTSS. We find that our proposed scheme can perform well under a wide variety of practical circumstances, and provides efficient and high performance intra-piconet and inter-piconet communications.

Keywords: Bluetooth, scatternets, ad hoc routing, time-slots scheduling.
\end{abstract}

\section{INTRODUCTION}

Bluetooth [3] is an important and increasingly popular wireless communication standard, and in recent years a large number of Bluetooth products have been introduced commercially. Its chief advantages are small and low power consumption, which make the technology an attractive choice when setting up short-range wireless ad hoc networks. A typical Bluetooth network, known as a piconet, consists of one master and seven slaves. When several Bluetooth networks interconnect through a number of bridge nodes, they form a scatternet. However, no routing protocol has yet been defined for Bluetooth scatternets. Meanwhile, because of its small size and low cost, Bluetooth is widely "pervasive", in the sense that even cheap electrical appliances will be expected to become Bluetooth enabled. If so, scatternets consisting of several piconets will exist even in a home environment. Thus, there is a pressing need to develop an efficient ad hoc routing algorithm [4] to make communication possible between distant devices.

This study proposes an integrated ad hoc routing and time- slot scheduling (IARTSS) scheme to address the problem of ad hoc routing in Bluetooth networks. We have built a comprehensive Bluetooth simulator and performed extensive simulations to evaluate the proposed IARTSS. We find that our proposed scheme can perform well under a wide variety of practical circumstances, and provides efficient and high performance intra-piconet and interpiconet communications.

\section{Overview of the Proposed Integrated SCHEME}

Our proposed scheme contains four main mechanisms to address the different facets of the problem, namely Compensation-Based Time-Slot Assignment (CTSA), Traffic Differentiation Queueing (TDQ), Adaptive Master-Slave Switching (AMSS), and an Enhanced AODV algorithm [1] for ad hoc routing. Due to space limitations, it is not possible for us to describe in detail the four mechanisms. The reader is referred to the on-line full report [2] for the detailed information. We only provide highlights in the following.

Compensation-Based Time-Slot Assignment (CTSA) is a scheduling algorithm tailor-made for Bluetooth. With the formation of scatternet, some nodes have to take up the role of routers or bridges to interconnect different piconets together. As Bluetooth uses a frequency hopping scheme, a node cannot listen to two piconets simultaneously. As a result, it can only synchronize and participate in one piconet at a time. This implies that these bridge nodes have to multiplex their time among different piconets. However, as Bluetooth adopts a centralized polling scheme for time slots allocations, these nodes have to compete for time slots with all the other nodes. when they switch back to another piconet. Consequently, the resources acquired by these nodes from the piconet are fewer. In a scatternet environment, traffic routing across the network can be abundant. The bridge nodes play an important role in rout- 
ing packets across different piconets and they become the bottlenecks of the system. Most of the existing scheduling algorithms allocate time slots according to the loads of the queues, and if we apply them to Bluetooth directly, we may have ignored the bridging and uplink traffic problems. CTSA is an advanced scheduling scheme for Bluetooth. It exploits the properties of the absence of bridge nodes to calculate the time slots allocation for each nodes more effectively.

Traffic Differentiation Queueing (TDQ) is a technique to separate different traffic to different queues in Bluetooth's link layer. Traffic is differentiated as "self traffic" and "forward traffic" in this scheme and they are put into two different queues. Those being generated by the Bluetooth host itself are classified as "self traffic" while those being forwarded to it are classified as "forward traffic". The Bluetooth link layer serves these queues based on an adaptive ratio manner. This scheme helps routing packets across scatternet more efficiently and can improve the throughput and delay in a large extent.

Adaptive Master-Slave Switching (AMSS) is a hold time calculation scheme between bridge nodes, which decides the duration for a node to stay in a piconet. As bridge nodes in Bluetooth network need to multiplex their time to different piconets in a scatternet, the time spent on a piconet greatly affects the overall system performances. Hence, an adaptive calculation of the hold time is required. AMSS addresses this problem and uses an adaptive method based on queue lengths and utilization ratio to determine the hold time for a piconet. This scheme allocates time between piconets in a more effective way.

Enhanced AODV for Ad Hoc Routing is a routing protocol developed to facilitate ad hoc routing in Bluetooth network. AODV is used because it is one of the most representative ad hoc routing protocols and it performs well in most of the wireless network environment. In our enhanced AODV routing protocol, we have modified AODV' for Bluetooth so that it becomes suitable for Bluetooth's narrow bandwidth and small packet size features. Our modifications are mainly about reducing the overheads in the AODV protocol, making the modified version more suitable for the Bluetooth environments.

\section{Simulation Results}

In our performance study, Bluetooth networks with a variable number of nodes are examined. The Bluetooth topology used is a Bluetooth scatternet with a maximum of three masters and two bridge nodes, and thus, restricting the number of piconets to be a maximum of three in our simulation environment. We also focus on the worst case scenario--seven slaves are in each piconet. For traf- fic load, we make use of the Constant Bit Rate (CBR) traffic and the Exponential (EXP) traffic source. The CBR source generates 160 bits regularly in $20 \mathrm{msec}$ time intervals, making the data rate $8 \mathrm{kbps}$. The EXP source is based on Poisson arrivals and has a data rate of $56 \mathrm{kbps}$. We vary the traffic load also by using different number of traffic sources in the scatternet: light load-2 CBR and 2 EXP, medium load-4 CBR and 4 EXP, and heavy load8 CBR and 8 EXP. To compare our IARTSS scheme with other practical approaches, we also simulate three other different schemes: round-robin (RR) time-slot scheduling with original AODV, deficit round-robin (DRR) time-slot scheduling with original AODV with two different deficit counter calculations [2]. The results are shown in Figures 1 to 5 .

\section{REMARKS ON THE RESULTS}

We have studied the performance of IARTSS and compared the results with two major scheduling schemes, which are the round robin algorithm and the deficit round robin algorithm, respectively. Results show that IARTSS can perform well in many situations and improve the performances in scatternet environment. These results are promising and suggest that Bluetooth, despite of its complication in network structure and polling scheme, can support communications in a wider network.

IARTSS generally works better than the other algorithms. It is because with the proposed time-slot scheduling and routing mechanisms, the overheads involved in getting packets through different piconets are much reduced. DRR1 and DRR2 generally works poorly because they tend to favor downlink traffic and discriminate the low volume uplink traffic.

Bluetooth technology is a new technology which just starts to emerge. There are still many rooms for further study and insightful development. Our research is just one of the beginnings, and more research problems could be addressed and studied in the future. If the proposed methods are applied in the real Bluetooth networks, interference will be an important problem. It is because when many Bluetooth devices exist together, they may experience a large interference. Therefore, the major limitation will be the maximum number of Bluetooth devices that can exist together. The study of interference in Bluetooth network is thus an urgent need. Also IARTSS calculates time slot assignments and hold time in simple mathematics only. More stringent calculations can actually be applied to them for more accurate approximations of the problem. In this research, we enhance the AODV for Bluetooth as the routing protocol. However, research in developing a completely novel ad hoc routing for Bluetooth may be a 


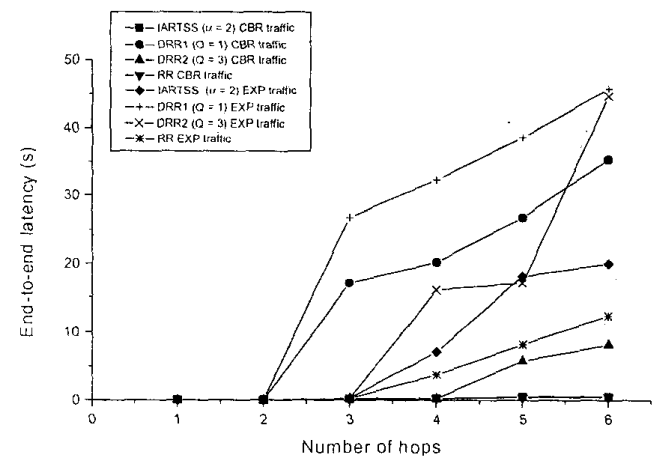

(a) end-to-end delay of scatternet traffic

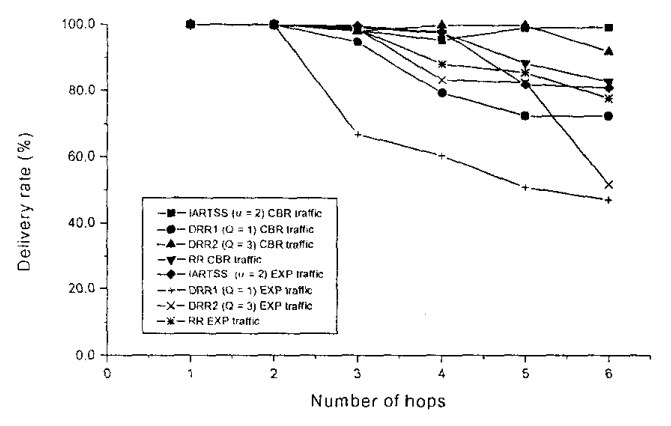

(b) delivery rate of scatternet traffic

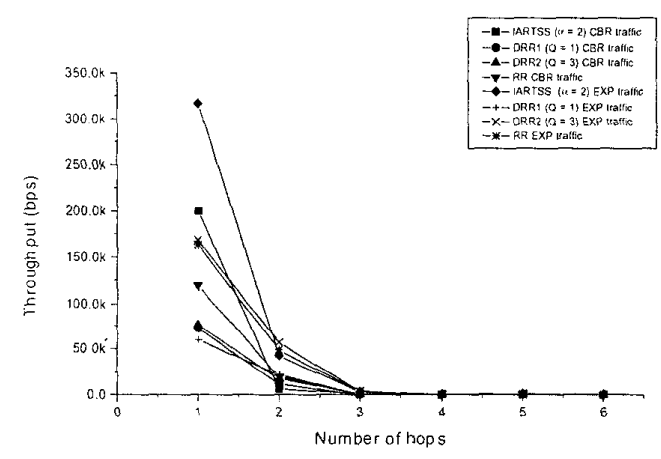

(c) throughput of scatternet traffic

Fig. 1. Comparison of different approaches using the DM3 packet type under the light load conditions.

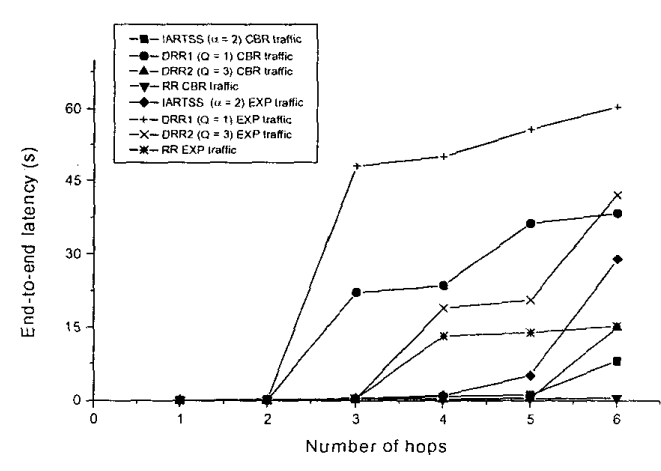

(a) end-to-end delay of scatternet traffic

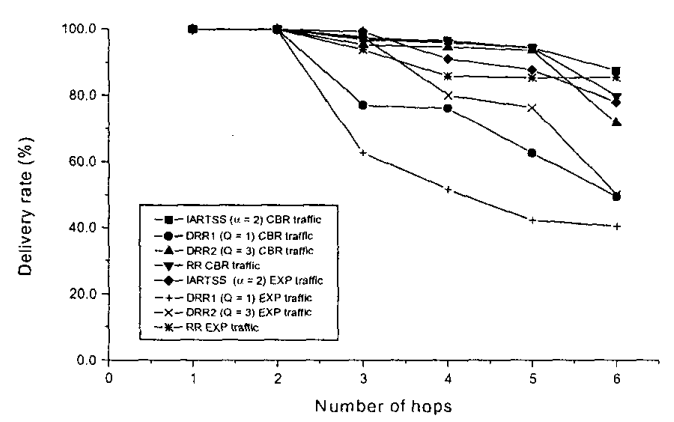

(b) delivery rate of scatternet traffic

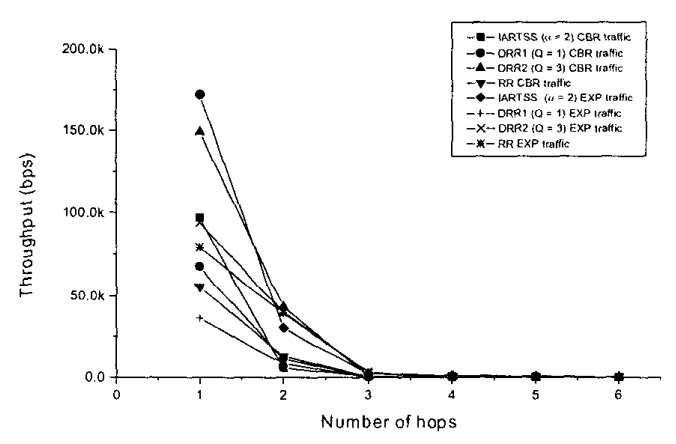

(c) throughput of scatternet traffic

Fig. 2. Comparison of different approaches using the DM3 packet type under the medium load conditions. 


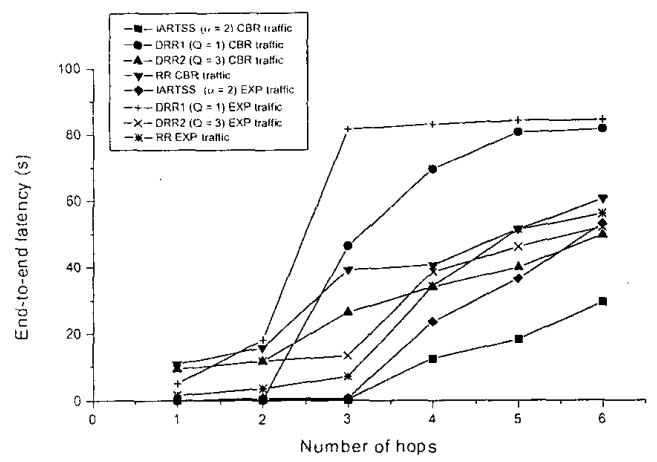

(a) end-to-end delay of scatternet traffic

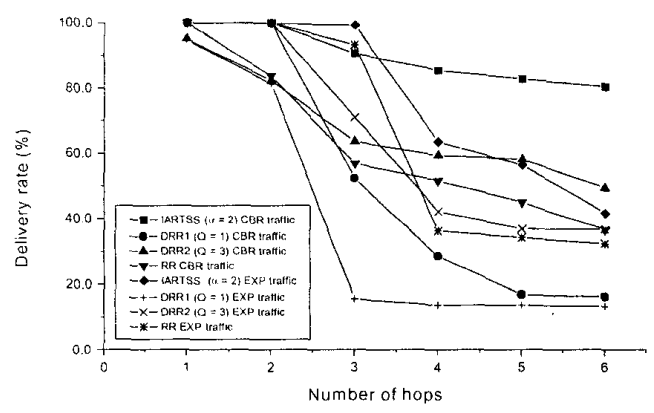

(b) delivery rate of scatternet traffic

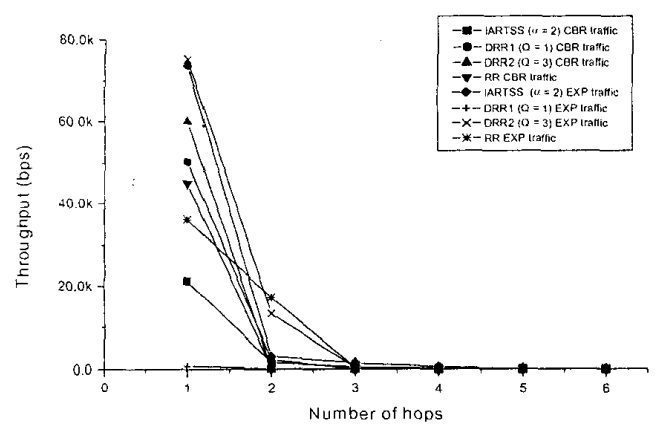

(c) throughput of scattemet traffic

Fig. 3. Comparison of different approaches using the DM3 packet type under the heavy load conditions.

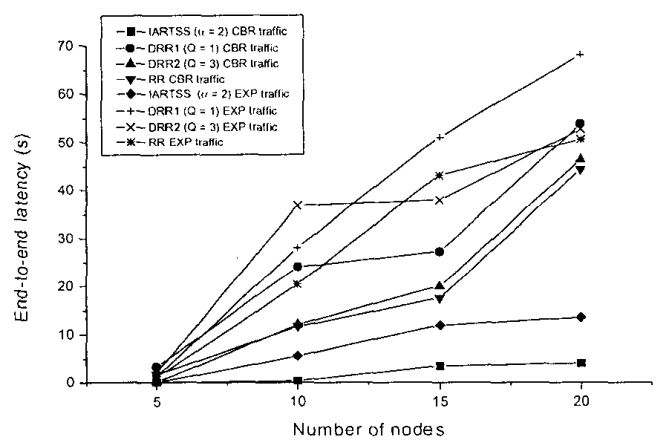

(a) end-to-end delay of scatternet traffic

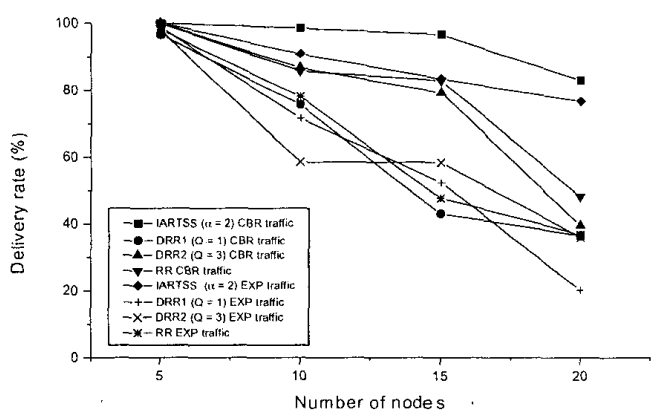

(b) delivery rate of scatternet traffic

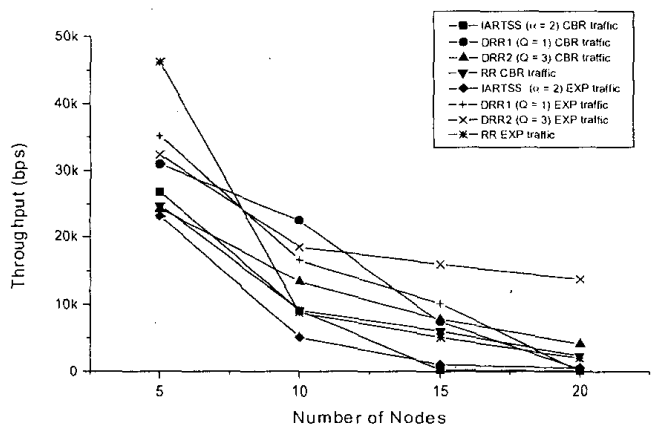

(c) throughput of scatternet traffic

Fig. 4. Comparison of different approaches using the DM3 packet type with various number of nodes in the scatternet. 


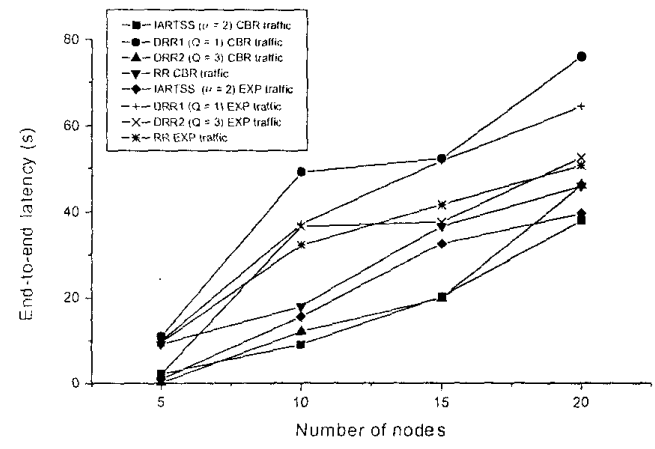

(a) end-to-end delay of scattemet traffic

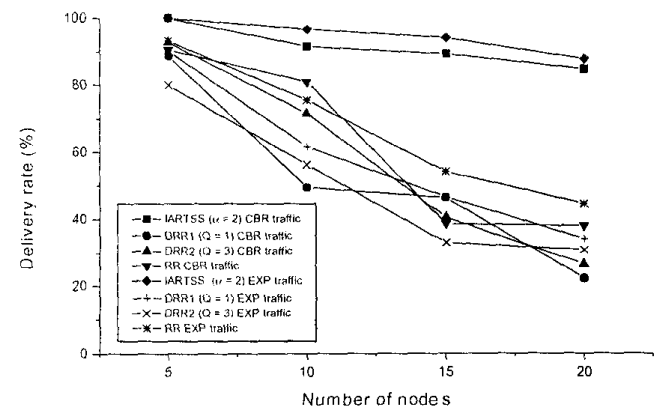

(b) delivery rate of scatternet traffic

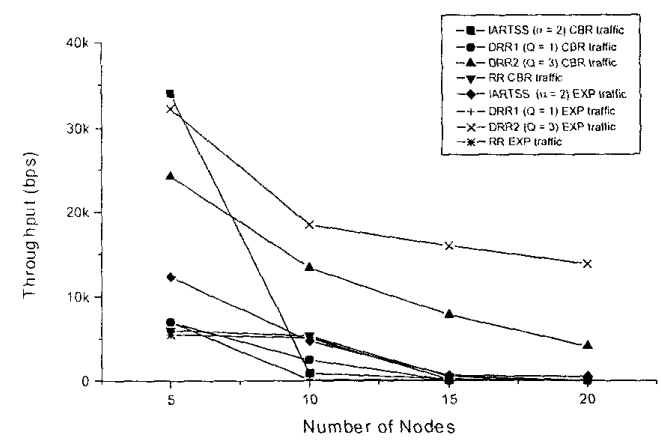

(c) throughput of scatternet traffic

Fig. 5. Comparison of different approaches using the DM5 packet type with various number of nodes in the scatternet. predominant factor to total success in this topic area.

\section{ACKNOWLEDGMENTS}

This research was supported by the Hong Kong Research Grants Council under project number HKU 7024/00E.

\section{REFERENCES}

[1] E. M. Royer and C. E. Perkins, "An Implementation Study of the AODV Routing Protocol," Proc. WCNC 2000, vol. 3, pp. 10031008, September 2000.

[2] L. L.-Y. Shek, Ad Hoc Routing and Time-Slot Scheduling in Bluetooth Networks, M.Phil. Thesis, The University of Hong Kong, December 2002 (http://www.eee.hku.hk/ ykwok/students/thesismphil-ShekLiza.pdf).

[3] The Official Bluetooth Web Site, http://www.bluetooh.com/dev/specifications.asp, 2002.

[4] C. K. Toh, Ad Hoc Mobile Wireless Networks: Protocols and Systems, Prentice Hall, 2002. 